\title{
Safety and efficacy of stereoelectroencephalography in pediatric focal epilepsy: a single-center experience
}

\author{
${ }^{*}$ Hannah E. Goldstein, MD, ${ }^{1}$ Brett E. Youngerman, MD, ${ }^{1}$ Belinda Shao, BA, ${ }^{2}$ Cigdem I. Akman, MD, ${ }^{3}$ \\ Arthur M. Mandel, MD, PhD, ${ }^{3}$ Danielle K. McBrian, MD, ${ }^{3}$ James J. Riviello, MD, ${ }^{4}$ \\ Sameer A. Sheth, MD, PhD, ${ }^{1}$ Guy M. McKhann, MD, ${ }^{1}$ and Neil A. Feldstein, MD² \\ 1Department of Neurological Surgery, Columbia University Medical Center, Columbia-Presbyterian, New York; ${ }^{2}$ Division of \\ Pediatric Neurosurgery, Department of Neurological Surgery, Children's Hospital of New York, Columbia-Presbyterian, New York; \\ ${ }^{3}$ Department of Neurology, Child Neurology Division, Children's Hospital of New York, Columbia-Presbyterian, New York, New \\ York; and ${ }^{4}$ Department of Neurology and Developmental Neuroscience, Texas Children's Hospital, Houston, Texas
}

OBJECTIVE Patients with medically refractory localization-related epilepsy (LRE) may be candidates for surgical intervention if the seizure onset zone (SOZ) can be well localized. Stereoelectroencephalography (SEEG) offers an attractive alternative to subdural grid and strip electrode implantation for seizure lateralization and localization; yet there are few series reporting the safety and efficacy of SEEG in pediatric patients.

METHODS The authors review their initial 3-year consecutive experience with SEEG in pediatric patients with LRE. SEEG coverage, SOZ localization, complications, and preliminary seizure outcomes following subsequent surgical treatments are assessed.

RESULTS Twenty-five pediatric patients underwent 30 SEEG implantations, with a total of 342 electrodes placed. Ten had prior resections or ablations. Seven had no MRI abnormalities, and 8 had multiple lesions on MRI. Based on preimplantation hypotheses, 7 investigations were extratemporal (ET), 1 was only temporal-limbic (TL), and 22 were combined ET/TL investigations. Fourteen patients underwent bilateral investigations. On average, patients were monitored for 8 days postimplant (range 3-19 days). Nearly all patients were discharged home on the day following electrode explantation.

There were no major complications. Minor complications included 1 electrode deflection into the subdural space, resulting in a minor asymptomatic extraaxial hemorrhage; and 1 in-house and 1 delayed electrode superficial scalp infection, both treated with local wound care and oral antibiotics.

SEEG localized the hypothetical SOZ in 23 of 25 patients (92\%). To date, 18 patients have undergone definitive surgical intervention. In 2 patients, SEEG localized the SOZ near eloquent cortex and subdural grids were used to further delineate the seizure focus relative to mapped motor function just prior to resection. At last follow-up (average 21 months), 8 of 15 patients with at least 6 months of follow-up (53\%) were Engel class I, and an additional 6 patients (40\%) were Engel class II or III. Only 1 patient was Engel class IV.

CONCLUSIONS SEEG is a safe and effective technique for invasive SOZ localization in medically refractory LRE in the pediatric population. SEEG permits bilateral and multilobar investigations while avoiding large craniotomies. It is conducive to deep, 3D, and perilesional investigations, particularly in cases of prior resections. Patients who are not found to have focally localizable seizures are spared craniotomies.

https://thejns.org/doi/abs/10.3171/2018.5.PEDS1856

KEYWORDS stereoelectroencephalography; SEEG; pediatric epilepsy; seizure monitoring

ABBREVIATIONS ATL = anterior temporal lobectomy; EEG = electroencephalography; ET = extratemporal; FCD = focal cortical dysplasia; $L R E=$ localization-related epilepsy; OR = operating room; SEEG = stereoelectroencephalography; SOZ = seizure onset zone; TCR = tailored cortical resection; TL = temporal-limbic. SUBMITTED January 26, 2018. ACCEPTED May 10, 2018.

INCLUDE WHEN CITING Published online July 20, 2018; DOI: 10.3171/2018.5.PEDS1856.

* G.M.M. and N.A.F. contributed equally to this work and share senior authorship. 
$\mathrm{E}$ PILEPSY affects approximately $1 \%$ of the population, with $30 \%-35 \%$ of patients having medically refractory epilepsy. A fraction of these patients are referred for surgical evaluation. Patients who are potential recipients of epilepsy surgery undergo extensive noninvasive workup to localize their seizure onset zone (SOZ). In addition to analysis of seizure semiology and continuous scalp electroencephalography (EEG) with video seizure monitoring, workup consists of various imaging modalities, as clinically indicated. In cases in which the SOZ still cannot be clearly localized, or there are conflicting data from the noninvasive workup, invasive seizure monitoring is frequently indicated to adequately lateralize and localize the SOZ.

In North America, invasive seizure monitoring has traditionally consisted of a large craniotomy to allow placement of a subdural grid over the surface of the brain and strip electrodes beneath the basal surfaces of the brain and interhemispherically. Subdural electrodes can be combined with a limited number of depth electrodes, if needed. Subdural monitoring is invasive and has many drawbacks. It is a large surgery with a significant major morbidity rate of $6 \%-25 \%$, even in experienced hands. Complications include CSF leaks; infections including meningitis and osteomyelitis, which may require premature termination of the implant; and loss of the bone flap. 1,2,5,8,24,26,30-33,36,38 The subdural electrodes create potentially symptomatic mass effect, particularly in the pediatric brain. The majority of children experience significant discomfort, typically lasting days to weeks. Traditionally, the size and magnitude of this surgery for SOZ localization has been a barrier for some parents, who may not be willing to put their children through this, especially without the guarantee that it will lead to a definitive surgical procedure and seizure improvement.

Furthermore, if the patient's seizures have not been adequately lateralized due to rapid ictal spread, as particularly occurs in frontal lobe epilepsy, a subdural strip lateralizing surgery may be necessary prior to subdural implantation. Reoperative surgery in cases in which prior surgery did not cure a patient's epilepsy can be technically challenging and more dangerous to the patient. Because subdural monitoring is a $2 \mathrm{D}$ procedure that records from the brain surface, these procedures have difficulty adequately recording deep surfaces such as the insula and cingulate gyrus, deep areas of focal cortical dysplasia (FCD), or deep margins of prior resection cavities. Given these limitations, epilepsy teams may be reluctant to recommend and patients' parents may not want to accept this approach. For these reasons, our center underwent a complete paradigm shift approximately 3 years ago, converting nearly all of our pediatric invasive seizure monitoring to stereoelectroencephalography (SEEG).

SEEG is not a new technique; it has been used in Europe, primarily in France and Italy, since the 1960s., 3,4,7, 9,15,17,18,25,27,29,34 However, this approach has only recently been incorporated into epilepsy surgery practice in North America. Gonzalez-Martinez and colleagues at the Cleveland Clinic have published a robust series of both adult and a subset of pediatric epilepsy surgery patients who underwent SEEG rather than subdural grid and strip in- vasive seizure monitoring. ${ }^{19-23}$ Our experience adds to the still sparse literature on the use of SEEG in pediatric patients, demonstrating safety and efficacy over our initial 3 -year experience.

\section{Methods \\ Patient Selection}

All pediatric patients (age $\leq 21$ years) with a diagnosis of medically refractory localization-related epilepsy (LRE) who were referred to the senior authors (N.A.F., G.M.M.) between June 2014 and November 2017 for surgical evaluation were presented at a multidisciplinary epilepsy conference. Any patient in whom it was believed that invasive seizure monitoring was necessary for better seizure-onset localization prior to definitive resection was considered for SEEG implantation. A total of 25 patients underwent SEEG implantation. Outpatient workup, epilepsy notes, and inpatient admissions were reviewed. Intraoperative data from the time of SEEG implantation and any operative complications were recorded. Epilepsy monitoring unit data were reviewed. The timing of SEEG explantation and data from follow-up clinic visits and subsequent epilepsy-related surgeries were gathered, and long-term seizure outcomes were evaluated when available.

All workup and surgical interventions were part of standard clinical patient care; no procedures were performed for research purposes. The study was reviewed and approved by the Columbia University Medical Center Institutional Review Board.

\section{SEEG Implantation and Monitoring}

All patients undergo a contrast-enhanced brain MRI study as well as a contrast-enhanced volumetric head CT scan prior to SEEG implantation. No patients have cerebral angiography for planning, because vascular structures are well visualized by the combined MRI and CT imaging. In collaboration with the epileptologists, targets of interest for seizure recording are identified based on preimplantation hypotheses of possible location of the patient's SOZ. Electrode trajectories are planned, with the aid of inline or trajectory views on the planning software, to target the desired brain locations, while avoiding vasculature and minimizing sulcal crossings. Most trajectories are orthogonally placed within $5^{\circ}-10^{\circ}$ of perpendicular to the skull to minimize the risk of electrode deflection. However, oblique trajectories from the top of the skull are often used for desired targets such as orbitofrontal cortex, the insula, and parasagittal areas such as supplementary motor area, primary motor and sensory cortex, and the precuneus (Fig. 1C). Implantation for the first 9 procedures was done using the Cosman-Roberts-Wells frame and iPlannet software (Brainlab), adjusting the coordinates of the frame for each electrode trajectory. We then transitioned to the ROSA robot (ROSA Surgical Robot, Zimmer Biomet), which has become an integral part of the procedure itself. While this shift has not changed target planning, seizure recording, or overall SOZ localization, it has simplified the intraoperative workflow and decreased overall operating time per SEEG electrode.

Use of the ROSA robot allows for all imaging to be 

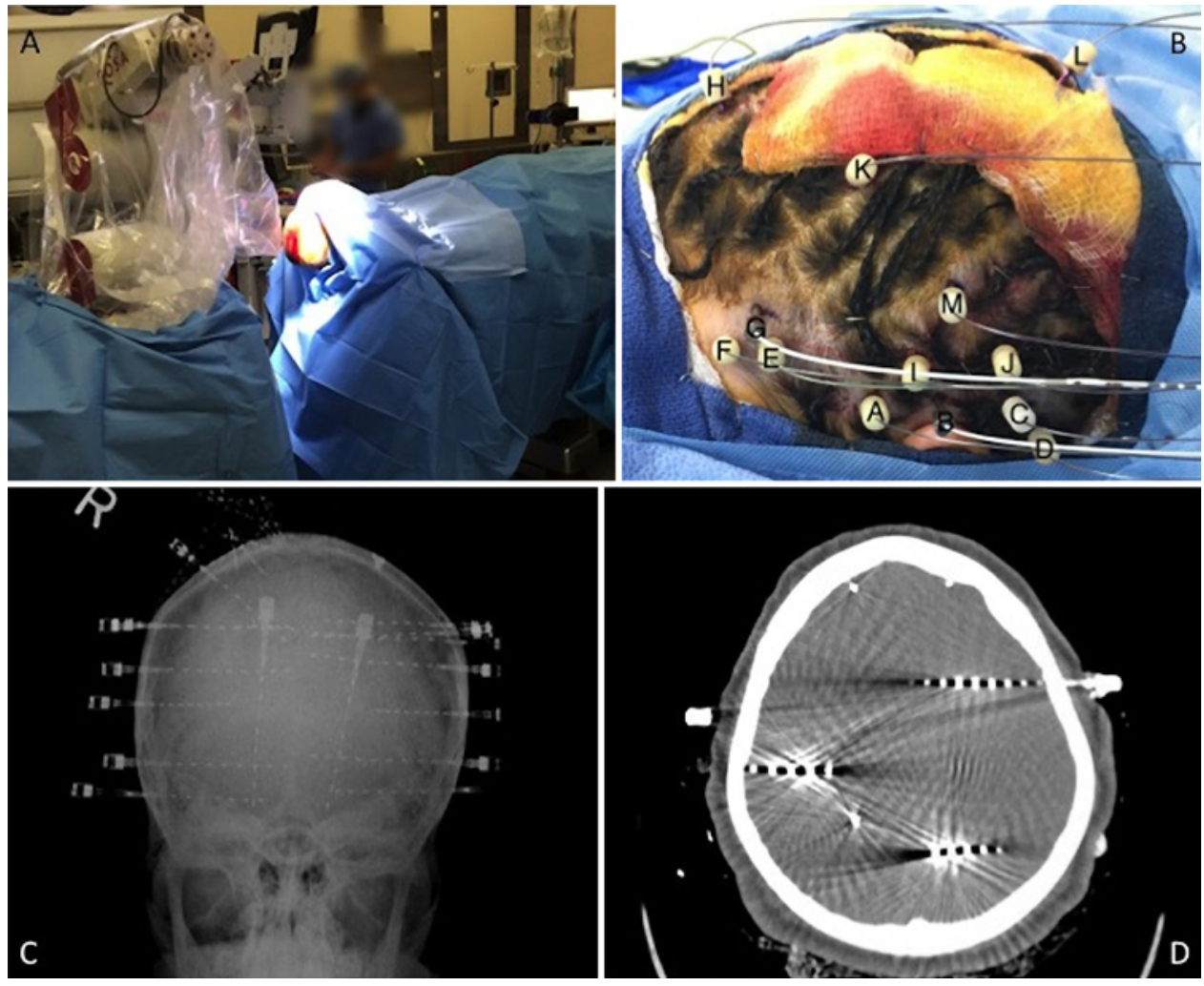

FIG. 1. Illustrative case. A: OR setup with ROSA robotic arm. B: Patient with implanted SEEG electrodes. C: Postoperative anteroposterior skull radiograph. D: Postoperative volumetric CT scan showing electrode trajectories. Figure is available in color online only.

obtained ahead of time. After the induction of general anesthesia, a stereotactic frame is attached to the patient's skull and their head is fixed to the robot by using a Mayfield adapter. With the ROSA robot, laser registration to the patient's volumetric CT scan 3D scalp reconstruction is carried out, and all electrode trajectory entry points are navigated to and marked on the scalp. A limited hair shave is performed, with preoperative box braiding encouraged in female patients to maximally preserve hair (Fig. 1B). Following sterile draping, SEEG electrodes are implanted in serial fashion (Fig. 1A). Both senior surgeons were present for all SEEG surgeries, with one focusing on stereotactic planning and trajectories and the other performing the electrode implantations.

For each electrode implantation, the robot is navigated to the desired stereotactic trajectory, from which it can only be navigated in the plane along the trajectory. The 3 - to 4-mm incision site is infiltrated with local anesthetic and incised with a No. 11 blade. An insulated probe is used to cauterize the deep scalp and periosteum. With the drill bit in place, the robot is moved closer to the patient's head, minimizing deflection. With a safety sleeve on the drill adjusted for measured bone thickness, the bone is drilled. A second, smaller, insulated probe is used to cauterize and penetrate the dura mater. Next, an anchor bolt of appropriate length is screwed into the bone down the stereotactic trajectory. The ROSA robot assists in calculating the depth from the anchor bolt to the planned electrode tip target.
An obturator needle is then passed through the anchor bolt to probe the electrode trajectory. An electrode of appropriate length is then passed to the target depth and tightened into place with the anchor bolt cap.

With experience, each electrode implantation takes approximately 5 minutes. At the end of the operation, all electrodes are connected, impedances are checked, and brief baseline EEG recordings are obtained. Anteroposterior and lateral skull radiographs are obtained to check electrode placement prior to removing the stereotactic frame. The patient is then awakened and taken to the imaging suite for a volumetric head CT scan (Fig. 1D). This CT scan is subsequently merged to the preoperative plan to confirm electrode locations relative to desired targets. All patients spend the first postoperative night in the pediatric intensive care unit, and are subsequently moved to the epilepsy monitoring unit on postoperative day 1 , assuming they are doing well clinically. Medication weaning is determined by the epileptologists.

Once adequate seizure data are obtained and electrode stimulation mapping is performed if indicated, patients return to the operating room (OR) for SEEG electrode explantation. Patients are generally discharged home the following morning. All patients are then re-presented in multidisciplinary epilepsy conferences, and are scheduled for surgery, if deemed appropriate. This turnaround time is usually at least 4-6 weeks, time enough to make sure that there are no areas of superficial scalp infection that 
would have to be transgressed during the resective craniotomy. However, given OR availability and family scheduling preferences, in our series definitive surgery was performed, on average, 10-13 weeks after explantation. Two patients had definitive surgery during the same hospital admission. In both cases, SEEG clearly delineated the SOZ after only a brief implantation. Given the clear SOZ, the families' desire to not return for a second procedure, combined with a very short SEEG implant time, the decision was made to proceed with resection 2-4 days after SEEG explantation.

\section{Results \\ Patient Demographics}

Twenty-five pediatric patients (age $\leq 21$ years) underwent a total of 30 SEEG implantation procedures between June 2014 and November 2017. All patients had a diagnosis of medically refractory LRE, without a clear SOZ, or conflicting evidence based on noninvasive workup. Once it was determined that invasive seizure monitoring was necessary, all but one patient who presented during this time were referred for SEEG implantation. The only patient to undergo initial grid placement had localization of his SOZ to the left temporal lobe based on noninvasive seizure workup. It was thought that he needed cortical language mapping in addition to SOZ localization, which was accomplished with a subdural grid and depth electrodes.

The average age at the time of SEEG implantation was 13 years old (range 5-21 years). Fifteen of the 25 patients $(60 \%)$ were female. Of the 5 patients who underwent 2 SEEG implantation procedures each, 2 had additional SEEG electrodes added during the same admission for more precise localization, based on SEEG monitoring data. One patient underwent reimplantation 2 months later in order to more precisely define the SOZ because very rapid spread was noted during the initial recording period, and the suspected SOZ was located close to primary motor cortex. One patient underwent a tailored cortical resection (TCR) based on the initial SEEG data, but continued to have seizures postoperatively, and so opted to undergo a second exploration with SEEG followed by a larger cortical resection. Now, more than 2 years from her second surgery, she remains seizure free. The fifth patient was recommended for resection following the first SEEG implantation. The parents decided to delay further intervention and were then lost to follow-up for approximately 2 years. When the patient re-presented, it was thought that further invasive seizure monitoring was necessary, because her seizures had changed clinically.

Including the patient who underwent a second SEEG procedure after initial SEEG followed by cortical resection, 10 patients had prior resections or laser ablations at the time of SEEG implantation. Seven patients had nonlesional MRI scans. Eight patients had multiple lesions seen on MRI-either multiple cortical tubers, multiple areas of FCD, encephalomalacia, or a focal finding in addition to a prior resection cavity (Fig. 2). Of the 30 SEEG implantation procedures performed, 7 were extratemporal (ET) investigations, 1 was limited to a temporal-limbic (TL) investigation, and 22 were combined ET/TL. There were

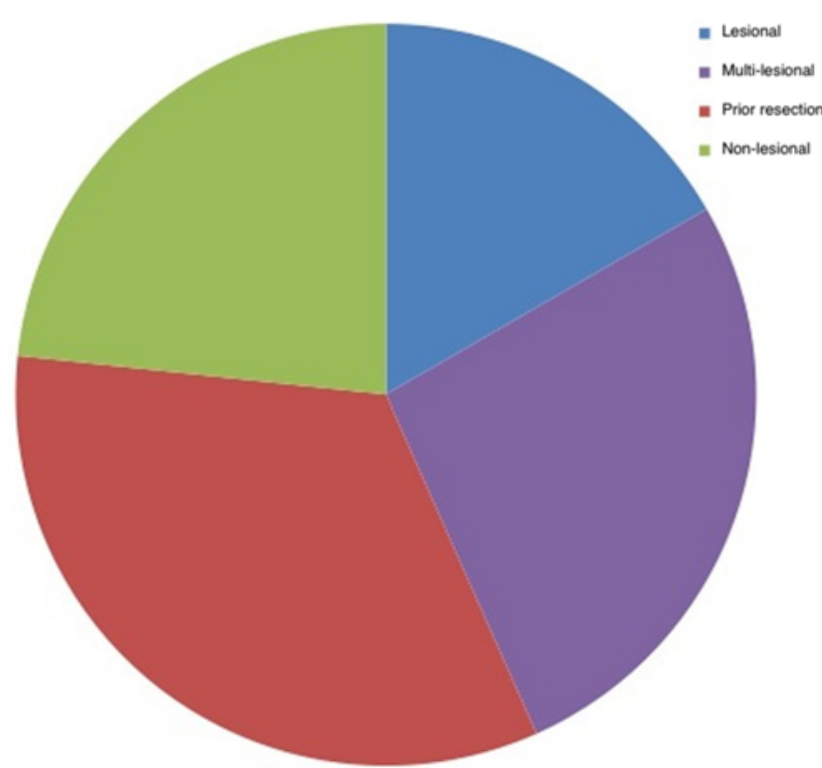

FIG. 2. Pie chart showing breakdown of preoperative MRI findings: 5 patients had a single lesion on their preoperative MRI; 8 patients had multiple lesions; 10 patients had a prior resection cavity; and 7 patients had nonlesional MRIs. Figure is available in color online only.

8 right-sided only, 8 left-sided only, and 14 bilateral investigations. Each patient had, on average, 11.4 electrodes implanted per procedure (range 4-18 electrodes), with a total of 342 SEEG electrodes implanted. Patients were monitored for, on average, 8 days postimplant (range 3-19 days), and the vast majority of patients were discharged home on postoperative day 1 following explantation (Table 1).

\section{Seizure Localization}

For SEEG to be efficacious in terms of identifying a patient's SOZ, it must be accurately hypothesis driven. The hypothetical SOZ was localized in 23 of the 25 patients (92\%) who underwent SEEG implantation. In one patient, who was known to have multiple different seizure semiologies, only 1 seizure was recorded over 19 days of monitoring. This patient pulled at her electrodes while agitated and disrupted the circuit connection. Given that all of the leads could no longer be monitored, they were explanted. The patient then had a spontaneous decrease in her seizure frequency ( 2 seizures per year), and the family has not pursued further surgical intervention. In the second patient in whom seizures were not localized, multiple seizures and seizure types were recorded during the SEEG monitoring, representing a probable multifocal epilepsy.

Of the 23 patients with localizable seizures, 18 have undergone definitive surgical intervention. One of the 5 patients who has not undergone definitive surgical intervention was found to have her SOZ located within primary motor cortex, and the family elected to undergo left vagal nerve stimulator placement. A second patient experienced spontaneous improvement in seizure frequency following SEEG and deferred further surgical intervention. The remaining 3 patients, the most recent ones undergoing SEEG in our cohort, are awaiting surgery. 
TABLE 1. Baseline characteristics of 25 patients with medically refractory LRE who underwent SEEG

\begin{tabular}{|c|c|c|c|c|c|c|c|c|c|c|}
\hline $\begin{array}{l}\text { Case } \\
\text { No. }\end{array}$ & $\begin{array}{l}\text { Age } \\
\text { (yrs) }\end{array}$ & Sex & Prior Surgery & MRI & $\begin{array}{l}\text { Scalp } \\
\text { EEG }\end{array}$ & $\begin{array}{l}\text { Side \& Location } \\
\text { of SEEG } \\
\text { Implantation }\end{array}$ & $\begin{array}{c}\text { No. of } \\
\text { SEEG } \\
\text { Electrodes }\end{array}$ & $\begin{array}{l}\text { Monitoring } \\
\text { Duration } \\
\text { (days) }\end{array}$ & $\begin{array}{l}\text { Localization of } \\
\text { SOZ }\end{array}$ & Complications \\
\hline 1 & 14 & M & None & NL & $\mathrm{BL} \mathrm{Fr}$ & BL ET/TL & 10 & 7 & Rt Fr & Trace hemorrhage \\
\hline 2 & 17 & $\mathrm{~F}$ & None & NL & $\mathrm{BL} \mathrm{Fr}$ & BL ET & 16 & 4 & Rt Fr & None \\
\hline 2 & 18 & $\mathrm{~F}$ & Rt Fr TCR & Resection & $\mathrm{Rt}>\mathrm{Lt} \mathrm{Fr}$ & BL ET & 9 & 4 & Rt Fr & None \\
\hline 3 & 20 & M & Lt tailored ATL & Resection & Lt T & Lt ET/TL & 8 & 4 & Lt T & None \\
\hline 4 & 8 & $\mathrm{~F}$ & None & $\mathrm{NL}$ & $\mathrm{BL}$ & BLET/TL & 16 & 6 & Lt Fr & None \\
\hline 5 & 16 & M & Rt TCR & Resection & Rt T & Rt ET/TL & 12 & 12 & Rt T & None \\
\hline 6 & 13 & $\mathrm{~F}$ & None & Lesional & Rt FT & Rt ET/TL & 14 & 10 & Rt FT & Superficial electrode infxn \\
\hline 7 & 5 & M & None & Multi & Lt Fr & Lt ET & 6 & 7 & Lt Fr & Trace IVH \\
\hline 8 & 20 & $\mathrm{~F}$ & None & Lesional & Rt FT & Rt TL & 10 & 4 & Rt T & None \\
\hline 9 & 12 & $\mathrm{~F}$ & Rt TCR & Resection & Rt TP & Rt ET/TL & 12 & 17 & \multirow{2}{*}{ Nonlocalizable } & \multirow{2}{*}{ None } \\
\hline 9 & 12 & $\mathrm{~F}$ & Rt TCR & Resection & Rt TP & Rt ET/TL & 4 & 7 & & \\
\hline 10 & 13 & M & None & Multi & Lt FTP & Lt ET/TL & 12 & 5 & $\begin{array}{l}\text { Nonlocalizable; } \\
\text { underwent rpt } \\
\text { SEEG }\end{array}$ & None \\
\hline 10 & 13 & M & None & Multi & Lt FTP & Lt ET/TL & 12 & 10 & LtP & None \\
\hline 11 & 15 & $\mathrm{~F}$ & Rt Fr LITT & Resection & Rt Fr & Rt ET & 7 & 3 & Rt Fr & Superficial electrode infxn \\
\hline 12 & 8 & M & None & Lesional & Lt FT & BL ET/TL & 12 & 15 & Lt Fr & Trace hemorrhage \\
\hline 12 & 10 & M & None & Lesional & Lt FT & Lt ET/TL & 12 & 6 & Lt Fr & None \\
\hline 13 & 14 & $\mathrm{~F}$ & None & Lesional & BL FPO & BL ET/TL & 14 & 19 & Nonlocalizable & $\begin{array}{l}\text { Trace hemorrhage; patient } \\
\text { broke leads }\end{array}$ \\
\hline 14 & 14 & $\mathrm{~F}$ & $\begin{array}{l}\text { Lt ATL + Fr } \\
\quad \text { lobectomy }\end{array}$ & Resection & $\mathrm{BLFT}$ & BL ET/TL & 12 & 5 & Lt FT (multifocal) & None \\
\hline 15 & 19 & M & Rt ATL & $\begin{array}{l}\text { Resection } \\
+ \text { lesion }\end{array}$ & Rt FTP & Rt ET/TL & 11 & 6 & Rt T & None \\
\hline 16 & 12 & $\mathrm{~F}$ & Rt Fr TCR & Resection & Rt FP & Rt ET & 5 & 7 & Rt Fr & None \\
\hline 17 & 20 & $\mathrm{~F}$ & None & $\mathrm{NL}$ & Lt FT & Lt ET/TL & 12 & 3 & Lt T & None \\
\hline 18 & 10 & M & None & NL & BL FTP & BLET/TL & 16 & 8 & Lt Fr & None \\
\hline 19 & 21 & $\mathrm{~F}$ & None & NL & BL FT & BL ET/TL & 14 & 12 & BL multifocal & None \\
\hline 20 & 7 & $\mathrm{~F}$ & None & NL & $\mathrm{Lt}>\mathrm{Rt} \mathrm{Fr}$ & BL ET & 14 & 3 & Lt Fr & None \\
\hline 21 & 11 & M & Lt Fr TCR & $\begin{array}{l}\text { Resection } \\
\quad+\text { lesion }\end{array}$ & $\mathrm{BL} F P$ & BL ET/TL & 10 & 7 & BL multifocal & None \\
\hline 22 & 9 & $\mathrm{~F}$ & None & Multi & Rt Fr & BL ET/TL & 13 & 7 & Rt Fr & None \\
\hline 23 & 15 & $\mathrm{~F}$ & None & Multi & Lt FTP & Lt ET/TL & 10 & 11 & itFr & None \\
\hline 23 & 15 & $\mathrm{~F}$ & None & Multi & Lt FTP & Lt ET/TL & 7 & 6 & Ltiा & None \\
\hline 24 & 7 & M & Lt TCR & Resection & Lt TP & BLET/TL & 14 & 6 & Lt TP (multifocal) & None \\
\hline 25 & 12 & $\mathrm{~F}$ & None & Multi & Lt Fr & BL ET & 18 & 9 & $\mathrm{Lt} \mathrm{Fr}$ & None \\
\hline
\end{tabular}

$\mathrm{BL}=$ bilateral; $\mathrm{FPO}=$ frontoparieto-occipital; $\mathrm{Fr}=$ frontal; $\mathrm{FT}=$ frontotemporal; $\mathrm{FTP}=$ frontotemporoparietal; infxn = infection; IVH = intraventricular hemorrhage; $\mathrm{LITT}=$ laser interstitial thermal therapy; multi = multiple lesions on $\mathrm{MRI} ; \mathrm{NL}=$ nonlesional; $\mathrm{P}=$ parietal; $r$ t $=$ repeat; $\mathrm{T}=$ temporal.

Of the 18 patients who did undergo surgical intervention after SEEG localization of SOZ, 15 underwent cortical resections: 5 underwent complete or partial frontal lobectomies; 2 underwent anterior temporal lobectomies (ATLs); 1 underwent a combined ATL with a modified frontal lobectomy for a large SOZ associated with FCD; and 7 underwent TCRs. One of the TCRs was done with the patient awake in order to conduct intraoperative functional testing. One patient underwent MRI-guided mesial temporal laser ablation. One patient who was found to have multifocal epilepsy underwent a complete corpus callosotomy. The final patient underwent a functional hemispherectomy for disconnection and has remained clinically seizure free. Pathological findings in resected specimens revealed 6 patients with FCD (FCD Ia, Ib, IIb, and Ic), 1 patient with microdysplasia, 5 patients with reactive gliosis, 1 patient with a known cortical tuber, 1 patient with a dysembryoplastic neuroepithelial tumor (WHO grade I), and 2 patients with no visualized abnormalities.

Excluding 1 patient who waited more than 1 year for 
TABLE 2. Outcomes of SEEG-guided surgical interventions in 15 patients with medically refractory LRE

\begin{tabular}{rrrrllll}
\hline $\begin{array}{c}\text { Case } \\
\text { No. }\end{array}$ & $\begin{array}{c}\text { Age } \\
\text { (yrs) }\end{array}$ & Sex & $\begin{array}{c}\text { Time to Surgical } \\
\text { Intervention (wks) }\end{array}$ & Type of Surgical Intervention & Pathology & $\begin{array}{c}\text { Length of } \\
\text { Follow-Up (mos) }\end{array}$ & $\begin{array}{c}\text { Seizure Outcome } \\
\text { (Engel class) }\end{array}$ \\
\hline 1 & 14 & M & 7 & Rt grids, Rt Fr lobectomy & Microdysplasia & 33 & I \\
\hline 2 & 18 & F & 2 & Rt Fr lobectomy & No abnormalities & 20 & I \\
\hline 3 & 20 & M & 11 & Lt ATL & Reactive gliosis & 25 & I \\
\hline 4 & 8 & F & 8 & Lt grids, Lt Fr TCR & FCD lb & 36 & IV \\
\hline 7 & 5 & M & 2 & Lt Fr TCR & FCD Ilb & 28 & II \\
\hline 8 & 20 & F & 14 & Rt ATL & Reactive gliosis & 33 & III \\
\hline 10 & 13 & M & 9 & Lt TCR & FCD la & 27 & III \\
\hline 11 & 15 & F & 1 & Rt TCR (awake) & Reactive gliosis & 15 & I \\
\hline 14 & 14 & F & 19 & Lt functional hemispherectomy & Reactive gliosis & 9 & I \\
\hline 15 & 19 & M & 10 & Rt TCR & FCD lb, Ic & 21 & II \\
\hline 17 & 20 & F & 8 & Lt laser ablation & NA & 21 & I \\
\hline 18 & 10 & M & 23 & Lt Fr lobectomy & No abnormalities & 15 & I \\
\hline 19 & 21 & F & 23 & Complete CC & NA & 11 & III \\
\hline 20 & 7 & F & 9 & Lt TCR & Reactive gliosis & 13 & III \\
\hline 22 & 9 & F & 8 & Rt Fr lobectomy & Cortical tuber & 8 & I \\
\hline
\end{tabular}

$\mathrm{CC}=$ corpus callosotomy; $\mathrm{NA}=$ not applicable (no biopsy taken).

definitive surgical intervention, the average time between SEEG explantation and resection was 10 weeks. In 2 patients, SEEG localized the SOZ near eloquent cortex, and a subdural grid implant was placed, followed by approximately 1 week of seizure monitoring and functional mapping prior to the definitive seizure resection. Of the 18 patients who have undergone definitive surgical intervention, the average active follow-up is 21 months, excluding 3 patients with less than 6 months of follow-up. At most recent follow-up, 8 of the 15 patients with at least 6 months of follow-up (53.3\%) are Engel class I (completely free of disabling seizures), and an additional 6 patients (40\%) are Engel class II or III (meaningful seizure improvement). Only 1 patient is Engel class IV, due to contralateral seizures, which began following a left frontal TCR. This patient had undergone bilateral SEEG monitoring, with all recorded seizures being left-sided in origin. Subsequently, genetic testing has revealed a mutation in the CHRNB2 gene for the beta- 2 cholinergic receptor, a mutation that is known to be associated with frontal lobe epilepsy syndromes (Table 2).

\section{Complications}

There were no major complications. Minor complications included 1 electrode that deflected into the subdural space with subtle, asymptomatic, extraaxial hemorrhage detected on a routine postoperative $\mathrm{CT}$ scan, and that did not require intervention. There were 3 other trace hemorrhages -2 in areas of prior resection cavities or encephalomalacia, and 1 instance of less than $1 \mathrm{ml}$ of intraventricular hemorrhage. All were asymptomatic and detected on surveillance postoperative CT scans, and none of them required intervention or interfered with seizure recording. There was 1 superficial scalp infection noted at the time of electrode removal, and 1 delayed superficial scalp infection found during routine office follow-up; both were treated with local wound care and oral antibiotics. One patient pulled at her leads, causing a disconnection from the monitoring circuit, but no intracranial injury. She underwent explantation the following day, because we were no longer able to record from all electrodes.

Our patients experienced no CSF leaks, no intracranial or bone infections, and no infections requiring wound washout or a return to the OR. Blood loss was minimal in all cases, eliminating the need for intra- or postoperative transfusions. Postoperative pain was also minimal. Some patients with predominantly temporal electrodes reported temporalis muscle pain with chewing, but compared to subdural electrode craniotomies the procedure is very well tolerated. Most patients are ambulating and eating the day after surgery, with minimal postoperative narcotic use. Patient activity and function are limited no more than they are with any continuous EEG monitoring, where the EEG electrodes are connected to a recording box.

\section{Discussion}

Although SEEG has been used in Europe for decades, it only began to gain favor in the US in the last half decade, in large part led by the Cleveland Clinic. ${ }^{19-23}$ Widespread use in the pediatric population has been slower to occur. We present our initial 3-year experience with SEEG used as the invasive monitoring technique of choice for nearly all pediatric patients with medically refractory LRE referred for invasive seizure monitoring.

Building off the work of others, ${ }^{19-23}$ we quickly modified and optimized our technique. We incorporated the ROSA robot as soon as fiscally possible, greatly decreasing operating time, and minimizing the risk of human error in electrode placement. We avoided cerebral angiography, instead relying on fused contrast-enhanced volumetric $\mathrm{MR}$ and $\mathrm{CT}$ images. We minimized hair removal and 
used box braids in females. All cases were staffed by two senior surgeons, with one focusing on stereotactic planning and the other dedicated to electrode implantation. We believe that our specific approach minimized our learning curve in adopting SEEG.

Previous papers have reported using SEEG in cases that are not amenable to standard strips and grids monitoring, or in particularly difficult-to-localize cases. ${ }^{20} \mathrm{In}$ our series, SEEG localized the hypothetical SOZ in all 7 patients with nonlesional MRI scans, and 4 of 7 (57.1\%) had Engel class I outcomes at an average of 21.3 months of followup. Similarly, SEEG ultimately localized the hypothetical SOZ in all 8 patients with multilesional MRI scans, with 6 of the 8 found to have a single seizure focus. However, we propose using SEEG as the initial invasive monitoring technique of choice for all surgical candidates with pediatric LRE. Not only does SEEG allow for bilateral and multilobar investigations in patients with multiple lesions or multiple seizure types, but it also allows for subcortical and mesial seizure foci monitoring in patients with presumed deep SOZs, and 3D and perilesional investigations in patients who continue to have seizures despite prior resections. Furthermore, patients found to have multiple SOZs precluding resection, or patients with lesions found to be in functionally eloquent cortex, are spared large craniotomies and the associated morbidity.

Perhaps most importantly for patients and their families, in our experience SEEG is much better tolerated than subdural implantation, with significantly reduced perioperative morbidity and pain. Despite almost 400 electrodes placed, we had no symptomatic bleeds and only 2 electrode superficial scalp infections. National and international studies have reported overall complication rates with SEEG of less than 1\%-3\%,7,10,28,37 This compares to complication rates generally reported as high as $6 \%-25 \%$ with subdural electrodes. $1,2,5,8,24,26,30-33,36,38$ Importantly, with SEEG, many of the risks of subdural invasive recording are avoided, significantly impacting the willingness of families to allow their young children to undergo the procedure. Furthermore, SEEG is very well tolerated by pediatric patients, making it an attractive alterative to a large invasive craniotomy for something that may prove to be only a diagnostic procedure.

Using SEEG for invasive seizure monitoring, we localized the hypothetical SOZ in 23 of 25 patients (92\%) in our series. One of the 23 patients with localizable seizures was found to have her SOZ within eloquent cortex, and we and the family decided against resection. A second patient had spontaneous improvement in seizure frequency and so is delaying surgical intervention. An additional 3 patients are currently awaiting surgery. Of the remaining 18 patients with SEEG-localized SOZs who have undergone definitive surgical intervention, 15 have at least 6 months of followup. Eight of 15 (53.3\%) have Engel class I outcomes, and an additional $6(40 \%)$ have Engel class II or III outcomes. Only 1 patient, who was later found to have a genetic mutation associated with frontal lobe epilepsy syndromes, has had no improvement in her seizures postresection (Engel class IV). Previous pediatric series looking at seizure outcomes after SEEG localization have reported Engel class I outcomes in $55.5 \%-67 \%$ of patients, ${ }^{11-14,20,22,23,35}$ with the discrepancy in outcomes largely attributable to the number of patients with nonlesional MRI scans included for evaluation. Our results, including Engel class I outcomes in 4 of the 7 patients (57.1\%) with nonlesional MRIs, are comparable to what has been previously reported..$^{10,20,23,30}$ Additionally, the predominance of pathological findings in surgical specimens showing FCD, considered to be one of the most frequent diagnoses associated with medically refractory LRE in the pediatric population, is consistent with what has been reported. ${ }^{6,16,22}$

One of the critiques of SEEG is that it is considered to be less conducive to functional mapping as compared to traditional strips and grids. In our series, there were 2 patients whose SOZ was found to be very close to eloquent cortex and in whom it was believed that further mapping was necessary prior to cortical resection. In both of these cases, the patients were brought back for targeted cortical subdural grid array placement and motor mapping just prior to their definitive resection. Depending on the age and cooperation of the patient, it is also reasonable in these cases to proceed directly to an awake craniotomy with mapping-based resection of the SOZ. Although some may argue that these patients could have been spared a surgery by undergoing strips and grids monitoring initially, in both cases the patients had nonlesional MRI scans and scalp EEG data indicative of bilateral seizure onset. Both patients underwent bilateral SEEG monitoring, and then were able to have much briefer and more targeted grid placement through a smaller craniotomy once the SOZ had been localized and a definitive surgical intervention had been planned.

Similarly, in both patients who required additional SEEG electrodes to be placed during their hospital admission, the SOZ was found to be at the edge of the area being monitored, and so additional electrodes were placed to expand the area of investigation and ensure that the entire SOZ was accurately captured. This can be done relatively easily, because each electrode is placed through its own stab incision and twist-drill burr hole, as opposed to needing to reopen an existing craniotomy or potentially turn a larger flap if this were found to be the case with a subdural grid.

\section{Conclusions}

We believe that SEEG is a superior alternative to subdural grid and strips for invasive seizure monitoring. SEEG is an appropriate first-line technique for all pediatric patients presenting with medically refractory LRE who require invasive intracranial monitoring, including children with nonlesional or multilesional MRIs, prior resection cavities, and multiple seizure semiologies. The main drawback to SEEG is that it is not as conducive to functional mapping. The appropriate surgical technique, of course, needs to be decided on a case-by-case basis. There are situations in which it may be rational and reasonable to implant a subdural array in a patient with suspected dominant temporal lobe or peri-Rolandic epilepsy. However, when the noninvasive seizure workup is not as clear, SEEG allows primary delineation of the SOZ and may avoid the need for a large craniotomy for subdural electrode implanta- 
tion. We have found that, when indicated, further functional mapping can be done either intraoperatively at the time of resection in older children or with a more focused cortical array implantation just prior to the planned resection. Additionally, as with any surgical technique, surgeon comfort, familiarity, and preference should also be taken into consideration. Overall, SEEG is a very safe technique and, when appropriately hypothesis driven, has a high rate of accuracy in terms of SOZ localization, with initial seizure outcomes comparable to those seen following other surgical interventions. We are continuing to follow these patients to determine the long-term outcomes after SEEGguided resection in the pediatric population with medically refractory localization-related epilepsy.

\section{References}

1. Adelson PD, O'Rourke DK, Albright AL: Chronic invasive monitoring for identifying seizure foci in children. Neurosurg Clin N Am 6:491-504, 1995

2. Arya R, Mangano FT, Horn PS, Holland KD, Rose DF, Glauser TA: Adverse events related to extraoperative invasive EEG monitoring with subdural grid electrodes: a systematic review and meta-analysis. Epilepsia 54:828-839, 2013

3. Bancaud J, Angelergues R, Bernouilli C, Bonis A, BordasFerrer M, Bresson M, et al: Functional stereotaxic exploration (SEEG) of epilepsy. Electroencephalogr Clin Neurophysiol 28:85-86, 1970

4. Barba C, Barbati G, Minotti L, Hoffmann D, Kahane P: Ictal clinical and scalp-EEG findings differentiating temporal lobe epilepsies from temporal 'plus' epilepsies. Brain 130:19571967, 2007

5. Bittar RG, Rosenfeld JV, Klug GL, Hopkins IJ, Harvey AS: Resective surgery in infants and young children with intractable epilepsy. J Clin Neurosci 9:142-146, 2002

6. Blumcke I, Spreafico R, Haaker G, Coras R, Kobow K, Bien $\mathrm{CG}$, et al: Histopathological findings in brain tissue obtained during epilepsy surgery. N Engl J Med 377:1648-1656, 2017

7. Bourdillon P, Ryvlin P, Isnard J, Montavont A, Catenoix H, Mauguière F, et al: Stereotactic electroencephalography is a safe procedure, including for insular implantations. World Neurosurg 99:353-361, 2017

8. Bruce DA, Bizzi JW: Surgical technique for the insertion of grids and strips for invasive monitoring in children with intractable epilepsy. Childs Nerv Syst 16:724-730, 2000

9. Budke M, Avecillas-Chasin JM, Villarejo F: Implantation of depth electrodes in children using VarioGuide ${ }^{\circledR}$ frameless navigation system: technical note. Oper Neurosurg (Hagerstown) [epub ahead of print], 2017

10. Cardinale F, Casaceli G, Raneri F, Miller J, Lo Russo G: Implantation of stereoelectroencephalography electrodes: a systematic review. J Clin Neurophysiol 33:490-502, 2016

11. Cossu M, Cardinale F, Castana L, Citterio A, Francione S, Tassi L, et al: Stereoelectroencephalography in the presurgical evaluation of focal epilepsy: a retrospective analysis of 215 procedures. Neurosurgery 57:706-718, 2005

12. Cossu M, Cardinale F, Colombo N, Mai R, Nobili L, Sartori I, et al: Stereoelectroencephalography in the presurgical evaluation of children with drug-resistant focal epilepsy. J Neurosurg 103 (4 Suppl):333-343, 2005

13. Cossu M, Lo Russo G, Francione S, Mai R, Nobili L, Sartori I, et al: Epilepsy surgery in children: results and predictors of outcome on seizures. Epilepsia 49:65-72, 2008

14. Cossu M, Schiariti M, Francione S, Fuschillo D, Gozzo F, Nobili L, et al: Stereoelectroencephalography in the presurgical evaluation of focal epilepsy in infancy and early childhood. J Neurosurg Pediatr 9:290-300, 2012
15. De Benedictis A, Trezza A, Carai A, Genovese E, Procaccini E, Messina R, et al: Robot-assisted procedures in pediatric neurosurgery. Neurosurg Focus 42(5):E7, 2017

16. Deleo F, Thom M, Concha L, Bernasconi A, Bernhardt BC, Bernasconi N: Histological and MRI markers of white matter damage in focal epilepsy. Epilepsy Res 140:29-38, 2018

17. Dorfmüller G, Ferrand-Sorbets S, Fohlen M, Bulteau C, Archambaud F, Delalande O, et al: Outcome of surgery in children with focal cortical dysplasia younger than 5 years explored by stereo-electroencephalography. Childs Nerv Syst 30:1875-1883, 2014

18. Freri E, Matricardi S, Gozzo F, Cossu M, Granata T, Tassi L: Perisylvian, including insular, childhood epilepsy: presurgical workup and surgical outcome. Epilepsia 58:1360-1369, 2017

19. González-Martínez J: Convergence of stereotactic surgery and epilepsy: the stereoelectroencephalography method. Neurosurgery 62 (Suppl 1):117-122, 2015

20. Gonzalez-Martinez J, Bulacio J, Alexopoulos A, Jehi L, Bingaman W, Najm I: Stereoelectroencephalography in the "difficult to localize" refractory focal epilepsy: early experience from a North American epilepsy center. Epilepsia 54:323-330, 2013

21. González-Martínez J, Bulacio J, Thompson S, Gale J, Smithason S, Najm I, et al: Technique, results, and complications related to robot-assisted stereoelectroencephalography. Neurosurgery 78:169-180, 2016

22. Gonzalez-Martinez J, Lachhwani D: Stereoelectroencephalography in children with cortical dysplasia: technique and results. Childs Nerv Syst 30:1853-1857, 2014

23. Gonzalez-Martinez J, Mullin J, Bulacio J, Gupta A, Enatsu R, Najm I, et al: Stereoelectroencephalography in children and adolescents with difficult-to-localize refractory focal epilepsy. Neurosurgery 75:258-268, 2014

24. Johnston JM Jr, Mangano FT, Ojemann JG, Park TS, Trevathan E, Smyth MD: Complications of invasive subdural electrode monitoring at St. Louis Children's Hospital, 19942005. J Neurosurg 105 (5 Suppl):343-347, 2006

25. Lagarde S, Bonini F, McGonigal A, Chauvel P, Gavaret M, Scavarda D, et al: Seizure-onset patterns in focal cortical dysplasia and neurodevelopmental tumors: relationship with surgical prognosis and neuropathologic subtypes. Epilepsia 57:1426-1435, 2016

26. Lee WS, Lee JK, Lee SA, Kang JK, Ko TS: Complications and results of subdural grid electrode implantation in epilepsy surgery. Surg Neurol 54:346-351, 2000

27. Liava A, Francione S, Tassi L, Lo Russo G, Cossu M, Mai $\mathrm{R}$, et al: Individually tailored extratemporal epilepsy surgery in children: anatomo-electro-clinical features and outcome predictors in a population of 53 cases. Epilepsy Behav 25:68-80, 2012

28. Mullin JP, Shriver M, Alomar SA, Najm I, Gonzalez-Martinez JA: 373 Is stereotacticelectroencephalography safe? A systematic review and meta-analysis of stereo-electroencephalography-related complications. Neurosurgery 63 (Suppl 1):211, 2016 (Abstract)

29. Pizzo F, Roehri N, Catenoix H, Medina S, McGonigal A, Giusiano B, et al: Epileptogenic networks in nodular heterotopia: a stereoelectroencephalography study. Epilepsia 58:2112-2123, 2017

30. Placantonakis DG, Shariff S, Lafaille F, Labar D, Harden C, Hosain S, et al: Bilateral intracranial electrodes for lateralizing intractable epilepsy: efficacy, risk, and outcome. Neurosurgery 66:274-283, 2010

31. Rolston JD, Englot DJ, Cornes S, Chang EF: Major and minor complications in extraoperative electrocorticography: a review of a national database. Epilepsy Res 122:26-29, 2016

32. Rolston JD, Ouyang D, Englot DJ, Wang DD, Chang EF: National trends and complication rates for invasive extraop- 
erative electrocorticography in the USA. J Clin Neurosci 22:823-827, 2015

33. Simon SL, Telfeian A, Duhaime AC: Complications of invasive monitoring used in intractable pediatric epilepsy. Pediatr Neurosurg 38:47-52, 2003

34. Talairach J, Bancaud J, Bonis A, Szikla G, Tournoux P: Functional stereotaxic exploration of epilepsy. Confin Neurol 22:328-331, 1962

35. Taussig D, Chipaux M, Lebas A, Fohlen M, Bulteau C, Ternier J, et al: Stereo-electroencephalography (SEEG) in 65 children: an effective and safe diagnostic method for pre-surgical diagnosis, independent of age. Epileptic Disord 16:280-295, 2014

36. Vale FL, Pollock G, Dionisio J, Benbadis SR, Tatum WO: Outcome and complications of chronically implanted subdural electrodes for the treatment of medically resistant epilepsy. Clin Neurol Neurosurg 115:985-990, 2013

37. van der Loo LE, Schijns O, Hoogland G, Colon AJ, Wagner GL, Dings JTA, et al: Methodology, outcome, safety and in vivo accuracy in traditional frame-based stereoelectroencephalography. Acta Neurochir (Wien) 159:1733-1746, 2017

38. Van Gompel JJ, Worrell GA, Bell ML, Patrick TA, Cascino GD, Raffel C, et al: Intracranial electroencephalography with subdural grid electrodes: techniques, complications, and outcomes. Neurosurgery 63:498-506, 2008

\section{Disclosures}

The authors report no conflict of interest concerning the materials or methods used in this study or the findings specified in this paper.

\section{Author Contributions}

Conception and design: Goldstein, Feldstein. Acquisition of data: all authors. Analysis and interpretation of data: Goldstein. Drafting the article: Goldstein. Critically revising the article: Goldstein, Akman, McBrian, McKhann, Feldstein. Reviewed submitted version of manuscript: all authors. Approved the final version of the manuscript on behalf of all authors: Goldstein. Study supervision: McKhann, Feldstein.

\section{Correspondence}

Hannah E. Goldstein: Columbia University/NewYork-Presbyterian Hospital, New York, NY. heg2117@columbia.edu. 